Supporting Information

\title{
Helicity sorting and optical resolution of functionalized single-walled carbon nanotubes and its optical properties
}

Yutaka Maeda, ${ }^{*}$ Yui Konno, Michio Yamada

Department of Chemistry, Tokyo Gakugei University, Koganei, Tokyo 184-8501, Japan

Contents

Experimental

Figure S1 (a) Absorption and (b, c) PL spectra of SWNTs and SWNTs-xylyl (for enantiomeric separation) dispersed in a $1 \mathrm{wt} \%$ SDBS $\mathrm{D}_{2} \mathrm{O}$ solution. (d-f) Raman spectra of SWNTs and SWNTsxylyl. Black: SWNTs (before functionalization). Red: SWNTs-xylyl.

Figure S2 SEM image of SWNTs-xylyl.

Figure S3 HPLC profile of SWNTs and gradient proportion of DOC (wt\%). Conditions: column, $\phi 10$ $\mathrm{mm} \times 200 \mathrm{~mm}$; eluent, $\mathrm{H}_{2} \mathrm{O}$ containing $0.5 \mathrm{wt} \% \mathrm{SDS}, 0.5 \mathrm{wt} \% \mathrm{SC}$, and $\mathrm{X} \mathrm{wt} \% \mathrm{DOC}$, where $\mathrm{X}$ corresponds to the values shown on the gradient; flow rate, $1 \mathrm{~mL} \mathrm{~min}^{-1}$; and detection, absorption at $280 \mathrm{~nm}$.

Figure S4 CD spectra of SWNTs (0.029), SWNTs (0.0315), SWNTs-xylyl (0.029), and SWNTs-xylyl (0.0315). The intensity was normalized using the $E_{22}$ absorbance at $\sim 570 \mathrm{~nm}$.

Figure S5 Absorption spectra of SWNTs and SWNTs-xylyl before separation (diluted 40-fold, black) and the separated SWNTs and SWNTs-xylyl samples obtained at retention times of 75-80 min (red), 105-110 min (yellow), 135-140 min (green), 165-170 min (blue), 195-200 min (purple), and 225-230 min (pink), where the numbers in parentheses represent the DOC concentrations during HPLC separation.

Figure S6 Absorption and PL spectra $(\lambda \mathrm{ex}=570 \mathrm{~nm})$ of (a and c) SWNTs $(0.0315)$ and $(\mathrm{b}$ and d) SWNTs-xylyl (0.0315) at various concentrations.

Fig. S7 (a) Absorption and (b, c) PL spectra of SWNTs and SWNTs-xylyl (for concentration quenching experiments) dispersed in a $1 \mathrm{wt} \%$ SDBS $\mathrm{D}_{2} \mathrm{O}$ solution. (d-f) Raman spectra of SWNTs and SWNTsxylyl. Black: SWNTs (before functionalization). Red: SWNTs-xylyl. 
Figure S8 HPLC profile of SWNTs and SWNTs-xylyl and gradient proportion of DOC (wt\%). Conditions: column, $\phi 10 \mathrm{~mm} \times 200 \mathrm{~mm}$; eluent, $\mathrm{H}_{2} \mathrm{O}$ containing $0.5 \mathrm{wt} \% \mathrm{SDS}, 0.5 \mathrm{wt} \% \mathrm{SC}$, and $\mathrm{X}$

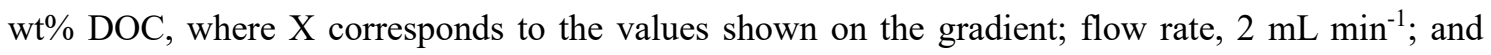
detection, absorption at $280 \mathrm{~nm}$.

Figure S9 Excitation spectra ( $\mathrm{E}_{11}{ }^{* *} \mathrm{PL}$ peak of H-SWNTs-xylyl (0.0315)). (a) $E_{22}$ absorption peak intensity: Black 0.094. Red 0.115. Yellow 0.145. Green 0.187. Blue: 0.235. Purple: 0.470.).

Figure S10 Fluorescence contour plots for H-SWNTs-xylyl (0.0315) at various concentration in 0.5 $\mathrm{wt} \% \mathrm{SC}+0.5 \mathrm{wt} \% \mathrm{SDS}+0.0315 \mathrm{wt} \%$ DOC aqueous solution.

Figure S11 PL spectra $\left(\lambda_{\text {ex }}=980 \mathrm{~nm}\right.$ ) of (a) SWNTs-xylyl and (b) H-SWNTs-xylyl (0.0315) at various concentrations (as indicated by the $E_{22}$ absorption peak intensities) measured at a 90 angle. (c) PL spectra $(\lambda e x=980 \mathrm{~nm})$ of H-SWNTs-xylyl $(0.0315)$ at various concentrations (as indicated by the $E_{22}$ absorption peak intensities) measured at a 22.5 angle.

Figure S12 PL spectra $\left(\lambda_{\mathrm{ex}}=580 \mathrm{~nm}\right)$ of (a) H-SWNTs (0.0315) and (b) H-SWNTs-xylyl (0.0315) at various concentrations (as indicated by the $E_{22}$ absorption peak intensities) in $0.5 \mathrm{wt} \% \mathrm{SC}+0.5 \mathrm{wt} \%$ SDS $+0.0315 \mathrm{wt} \%$ DOC aqueous solution measured at a $22.5^{\circ}$ angle relative to the excitation light.

Figure S13 PL spectra ( $E_{22}$ excitation) of H-SWNTs-xylyl (0.0315) (black line) at various concentrations (as indicated by the $E_{22}$ absorption peak intensities) at a $22.5^{\circ}$ angle relative to the excitation light and the corresponding Voigt curve fitting (colored areas). The FWHM values of $\mathrm{E}_{11}{ }^{*}$ PL peak (red) estimated from the peak fitting.

Figure S14 Bright line spectra of a xenon lamp as an excitation source for the PL measurement.

Table S1. $E_{22}$ absorption and $E_{22}$ CD peak wavelength of separated SWNTs. 
Naphthalene (300 mg, $2.34 \mathrm{mmol})$ and sodium $(156 \mathrm{mg}, 6.79 \mathrm{mmol})$ in a $200 \mathrm{~mL}$ heatdried three-necked round-bottom flask were placed under argon. Anhydrous tetrahydrofuran $(100 \mathrm{~mL})$ was then added to the flask and the contents were stirred for 30 min. A portion $(10.0 \mathrm{mg})$ of SWNTs in a $200 \mathrm{~mL}$ heat-dried three-necked round-bottom flask was placed under argon. The sodium naphthalenide solution was added to the SWNTs, which were then sonicated for $60 \mathrm{~min}$, and $2 \mathrm{~mL}$ of tetrahydrofuran solution of $371 \mathrm{mg}$ of 1,2-bis(bromomethyl)benzene $(1.405 \mathrm{mmol})$ was subsequently added to the mixture. The mixture was sonicated for $30 \mathrm{~min}$ and then quenched by the addition of 20 $\mathrm{mL}$ of dry ethanol. Afterwards, the reaction mixture was washed with water. The resulting suspended black solid was collected by means of filtration using a membrane filter (PTFE, $1.0 \mu \mathrm{m})$ and washed with tetrahydrofuran, methanol, and dichloromethane via the dispersion-filtration process. 

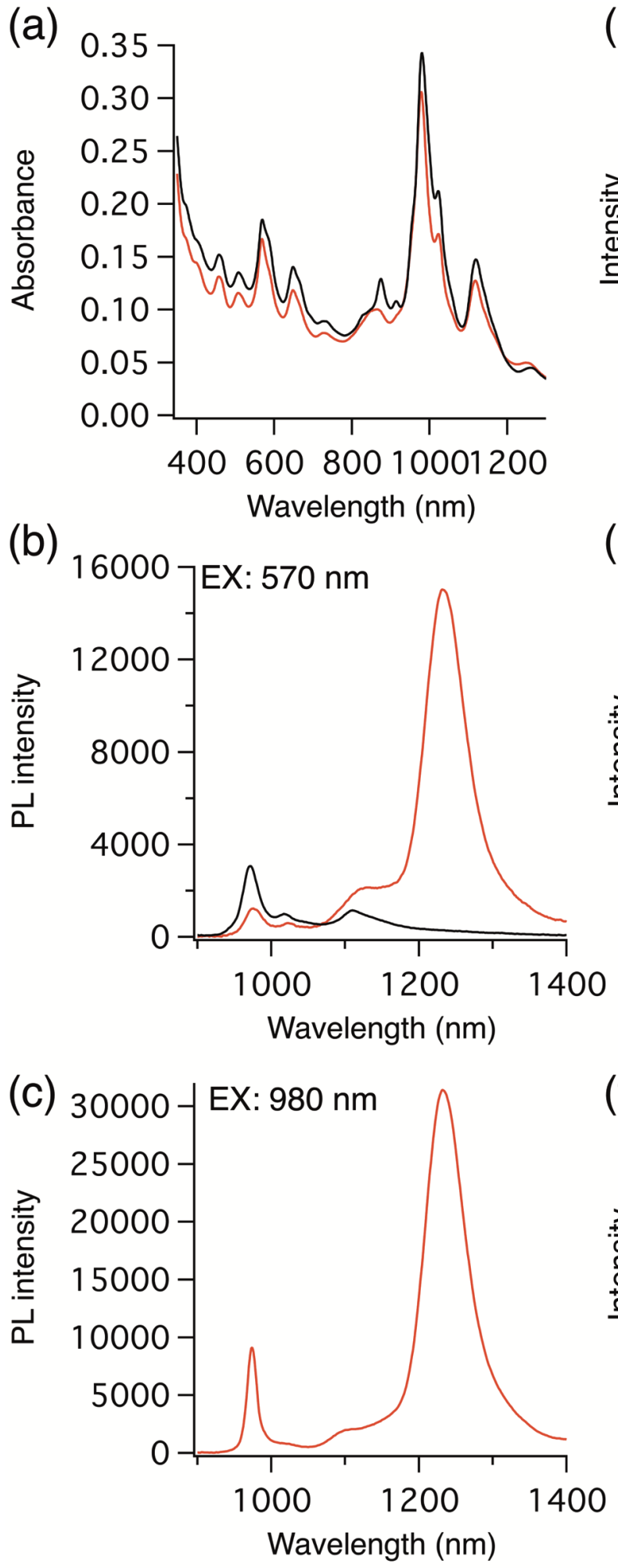
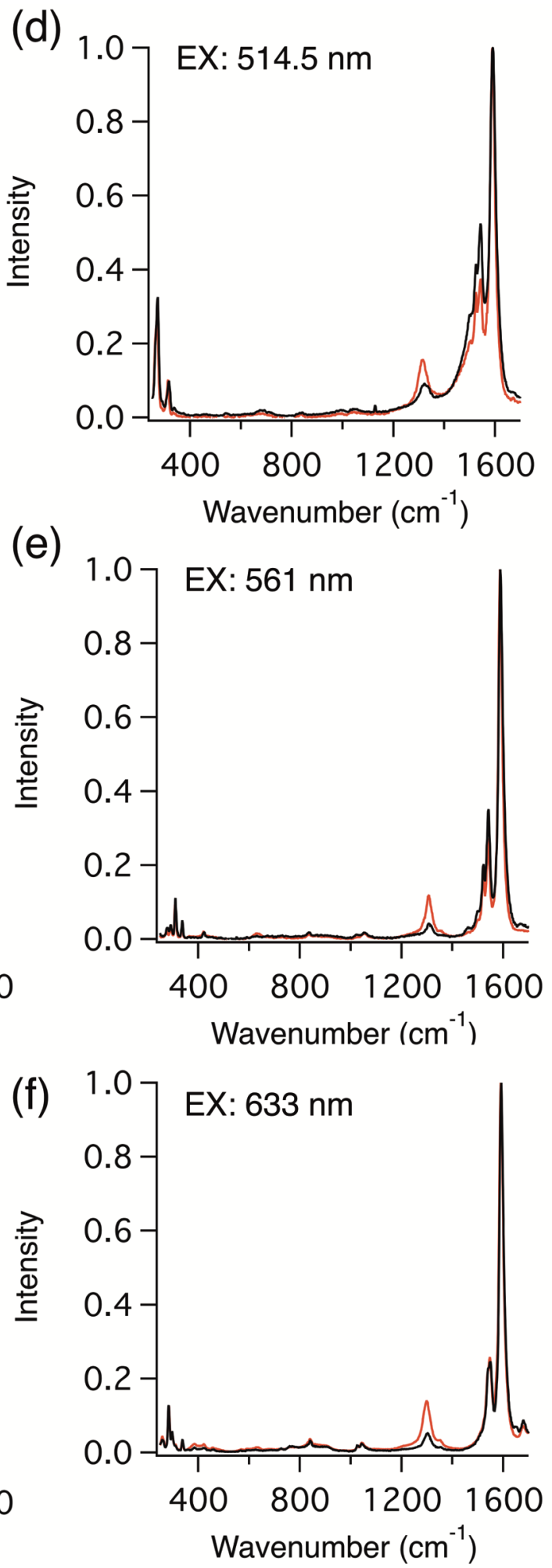

Figure S1 (a) Absorption and (b, c) PL spectra of SWNTs and SWNTs-xylyl (for enantiomeric separation) dispersed in a $1 \mathrm{wt} \%$ SDBS $\mathrm{D}_{2} \mathrm{O}$ solution. (d-f) Raman spectra of SWNTs and SWNTsxylyl. Black: SWNTs (before functionalization). Red: SWNTs-xylyl. 


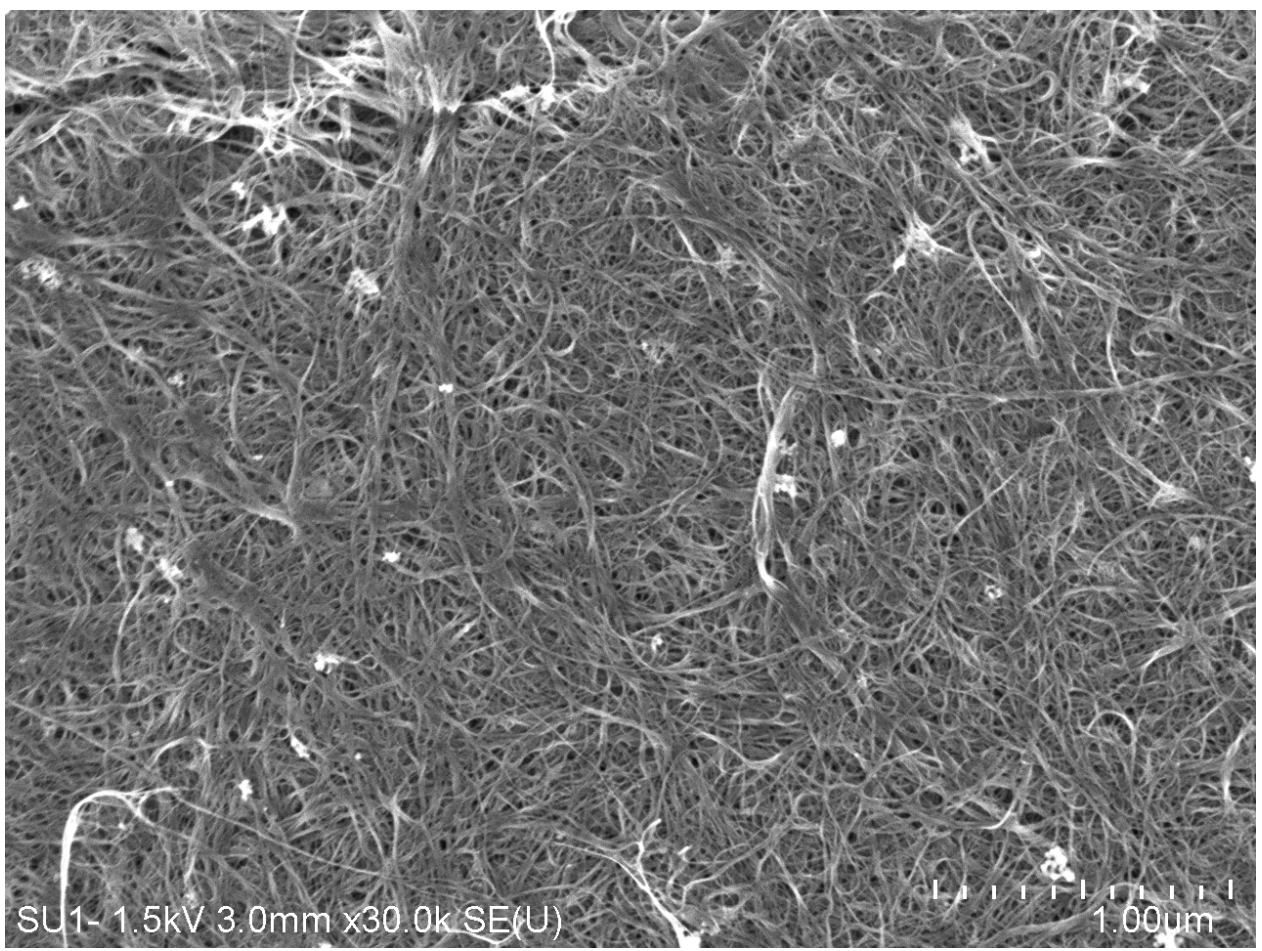

Figure S2 SEM image of SWNTs-xylyl.

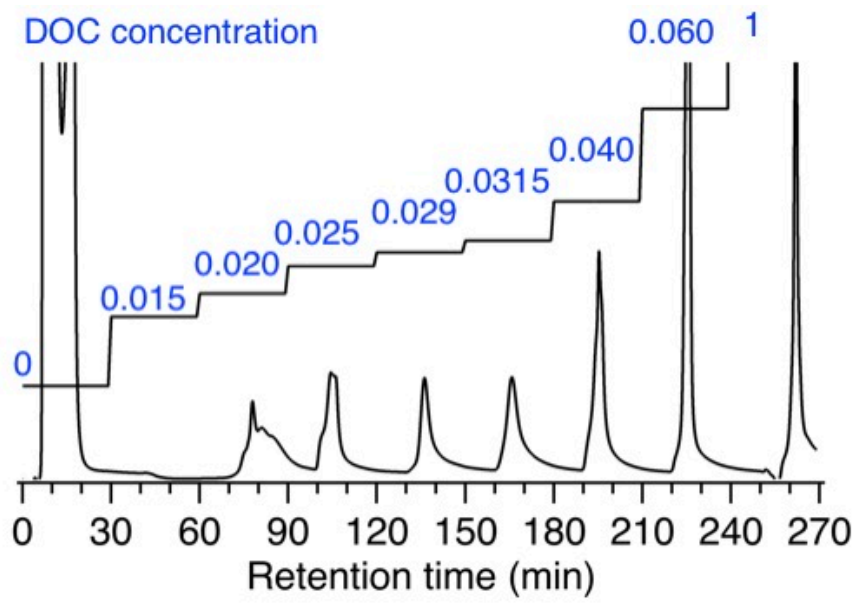

Figure S3 HPLC profile of SWNTs and gradient proportion of DOC (wt\%). Conditions: column, $\phi 10$ $\mathrm{mm} \times 200 \mathrm{~mm}$; eluent, $\mathrm{H}_{2} \mathrm{O}$ containing $0.5 \mathrm{wt} \% \mathrm{SDS}, 0.5 \mathrm{wt} \% \mathrm{SC}$, and $\mathrm{X} \mathrm{wt} \% \mathrm{DOC}$, where $\mathrm{X}$ corresponds to the values shown on the gradient; flow rate, $1 \mathrm{~mL} \mathrm{~min}{ }^{-1}$; and detection, absorption at $280 \mathrm{~nm}$. 

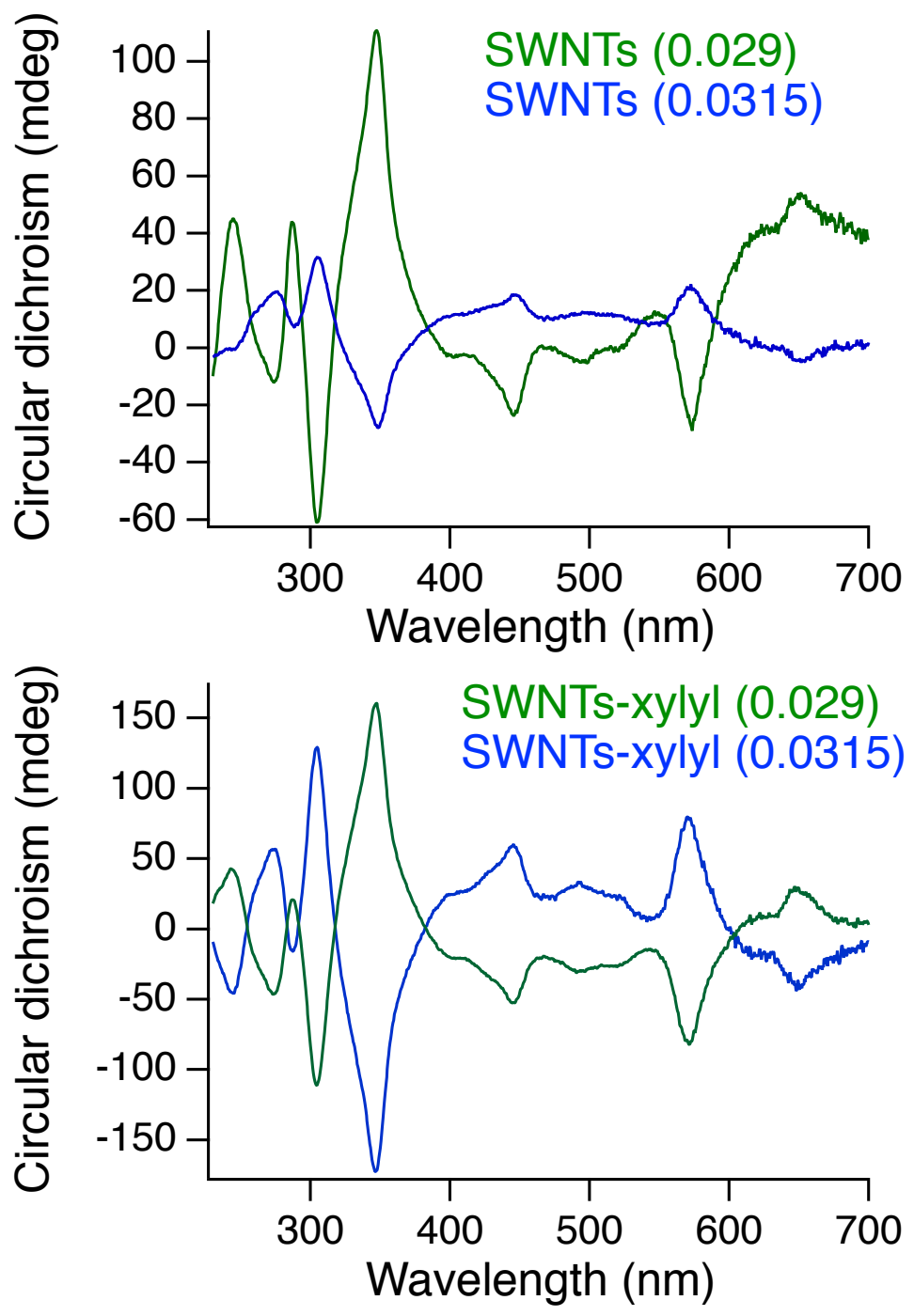

Figure S4 CD spectra of SWNTs (0.029), SWNTs (0.0315), SWNTs-xylyl (0.029), and SWNTs-xylyl $(0.0315)$. The intensity was normalized using the $E_{22}$ absorbance at $\sim 570 \mathrm{~nm}$. 

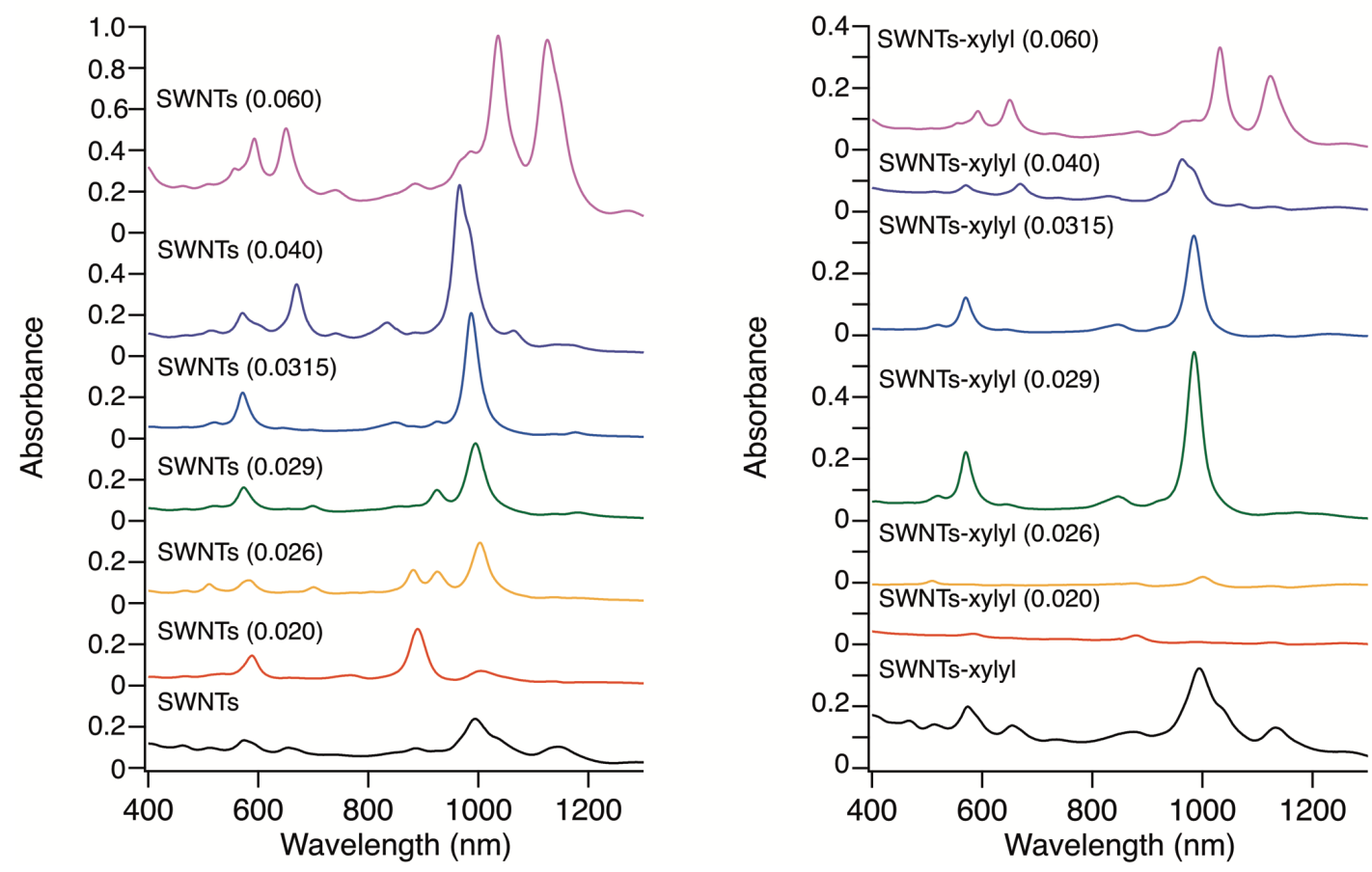

Figure S5 Absorption spectra of SWNTs and SWNTs-xylyl before separation (diluted 40-fold, black) and the separated SWNTs and SWNTs-xylyl samples obtained at retention times of 75-80 min (red), 105-110 min (yellow), 135-140 min (green), 165-170 min (blue), 195-200 min (purple), and 225-230 min (pink), where the numbers in parentheses represent the DOC concentrations during HPLC separation. 

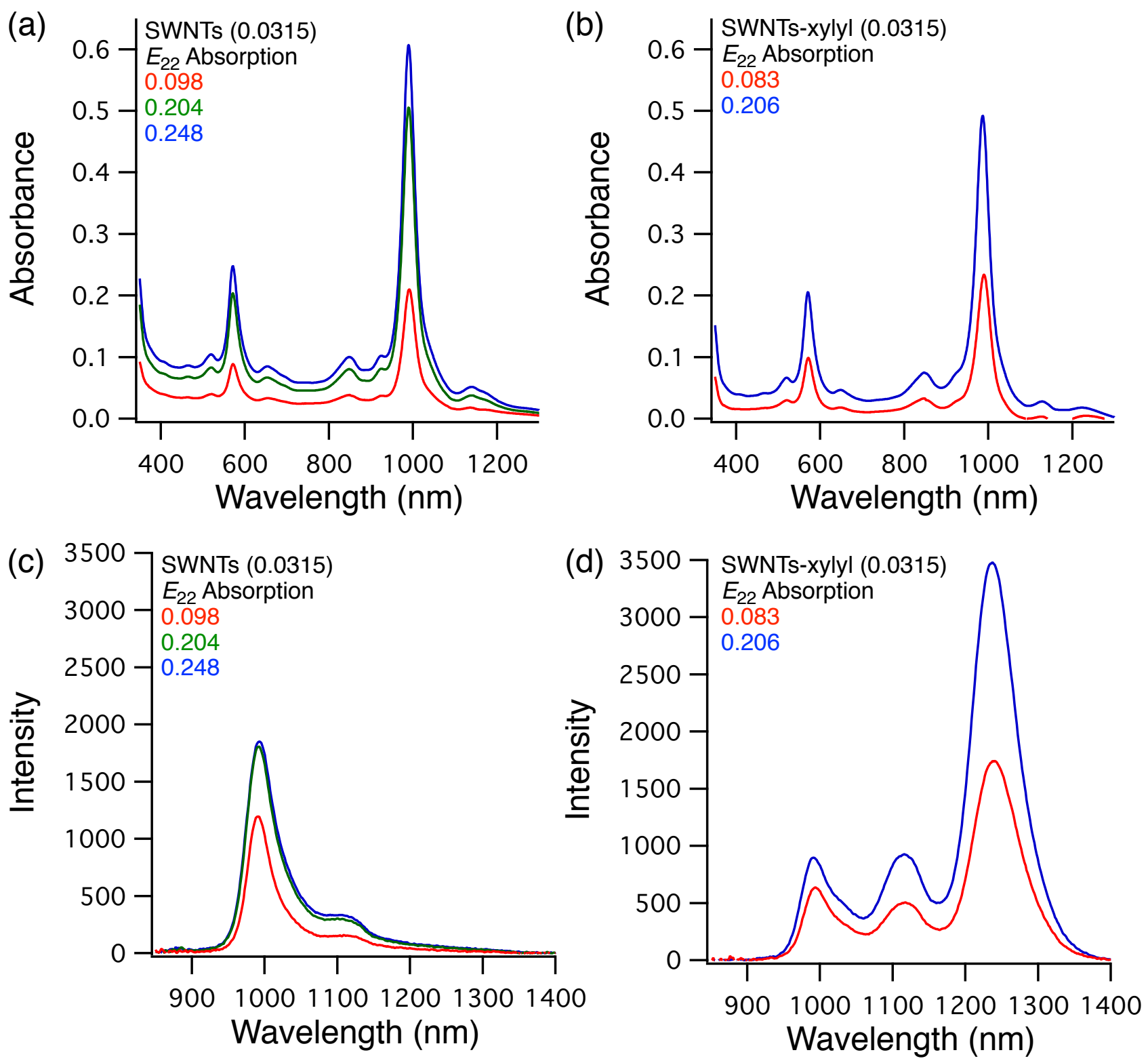

Figure S6 Absorption and PL spectra $(\lambda \mathrm{ex}=570 \mathrm{~nm})$ of (a and c) SWNTs $(0.0315)$ and $(\mathrm{b}$ and $\mathrm{d})$ SWNTs-xylyl (0.0315) at various concentrations. 

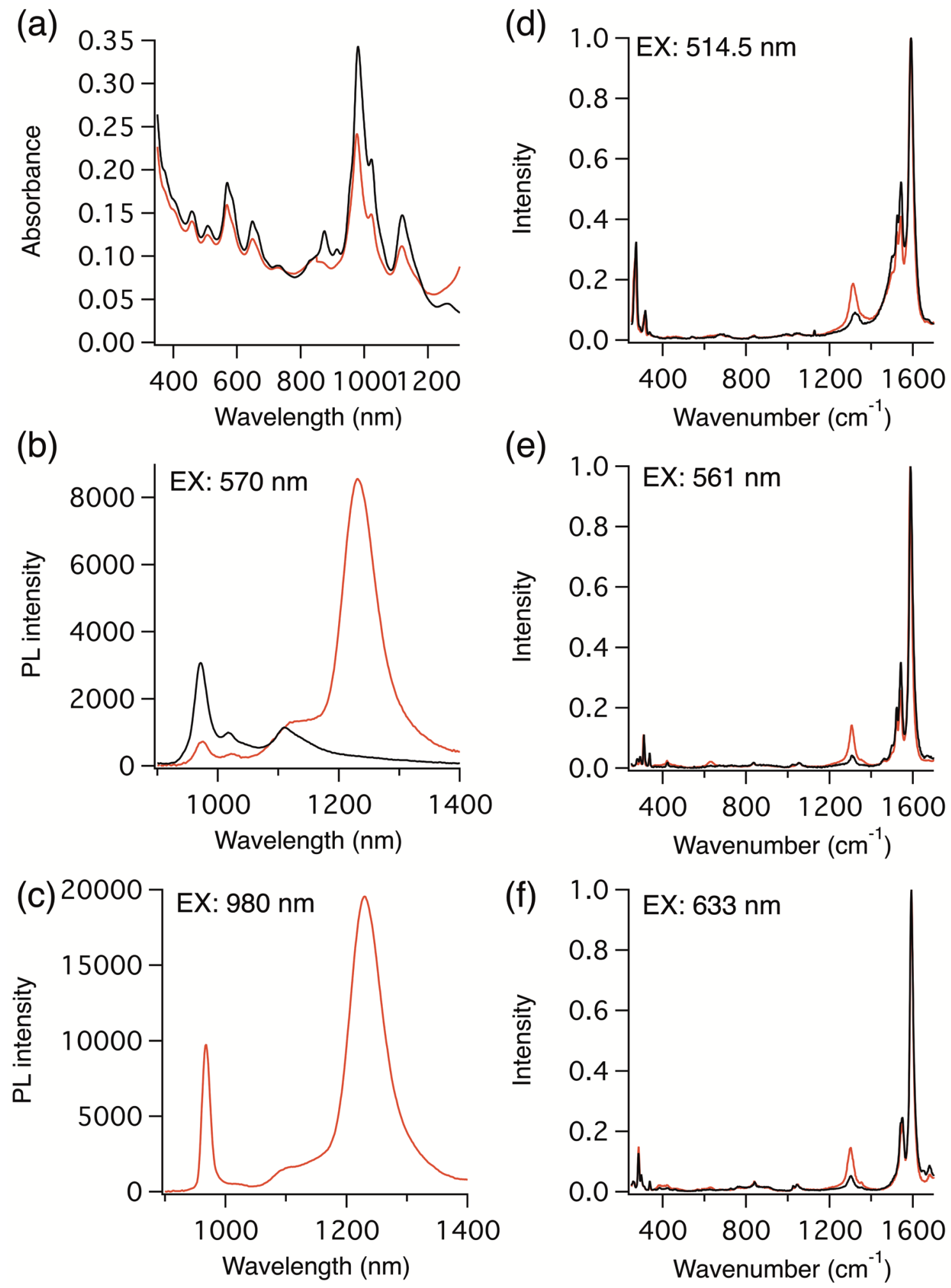

Figure S7 (a) Absorption and (b, c) PL spectra of SWNTs and SWNTs-xylyl (for concentration quenching experiments) dispersed in a $1 \mathrm{wt} \%$ SDBS $\mathrm{D}_{2} \mathrm{O}$ solution. (d-f) Raman spectra of SWNTs and SWNTs-xylyl. Black: SWNTs (before functionalization). Red: SWNTs-xylyl. 


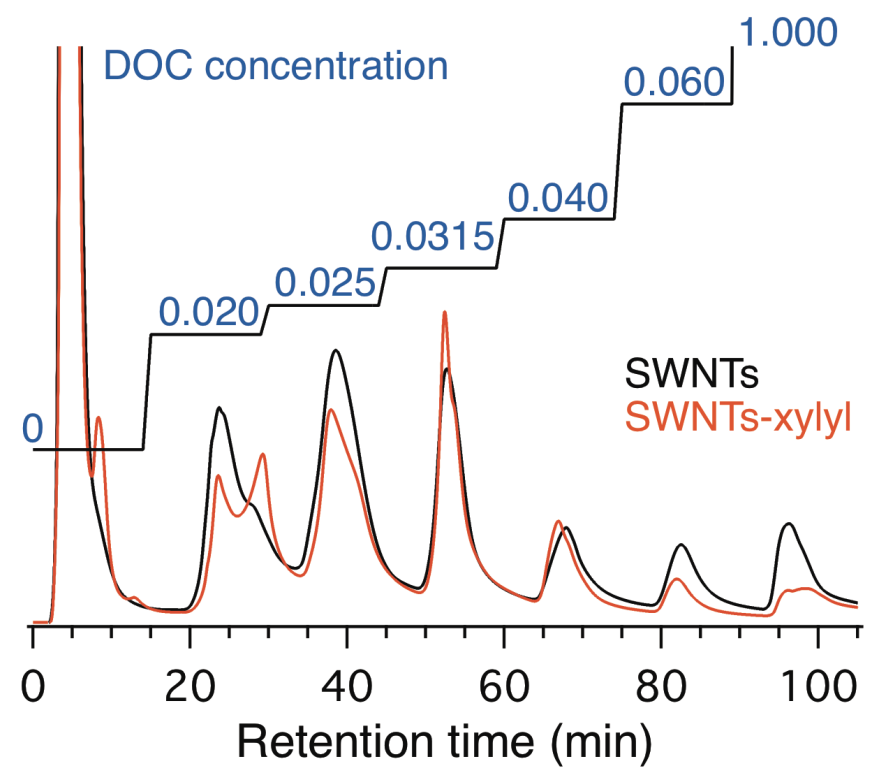

Figure S8 HPLC profile of SWNTs and SWNTs-xylyl and gradient proportion of DOC (wt\%). Conditions: column, $\phi 10 \mathrm{~mm} \times 200 \mathrm{~mm}$; eluent, $\mathrm{H}_{2} \mathrm{O}$ containing $0.5 \mathrm{wt} \% \mathrm{SDS}, 0.5 \mathrm{wt} \% \mathrm{SC}$, and $\mathrm{X}$

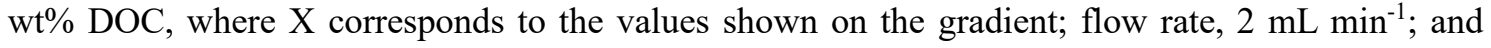
detection, absorption at $280 \mathrm{~nm}$.

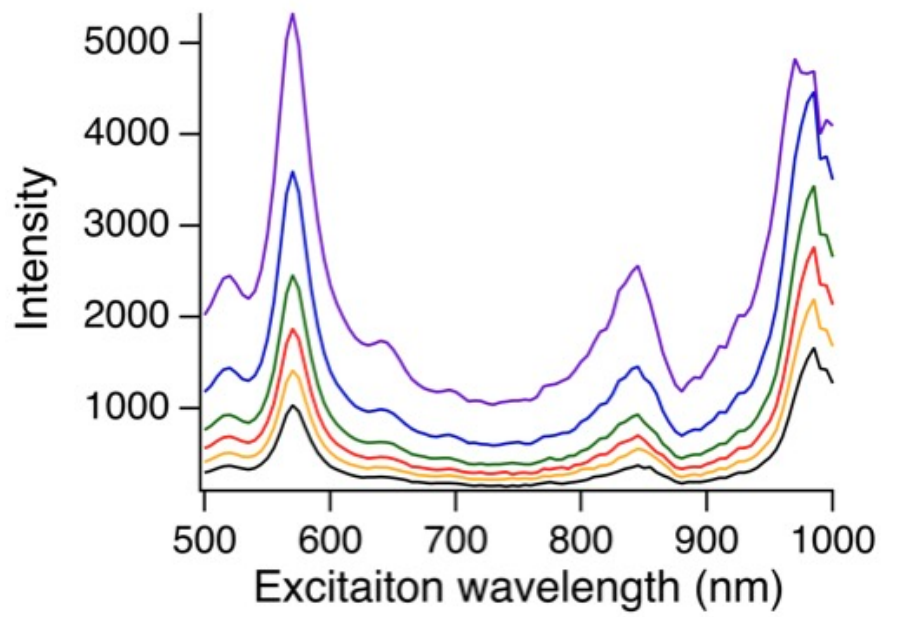

Figure S9 Excitation spectra ( $\mathrm{E}_{11}{ }^{* *}$ PL peak of H-SWNTs-xylyl (0.0315)). (a) $E_{22}$ absorption peak intensity: Black 0.094. Red 0.115. Yellow 0.145. Green 0.187. Blue: 0.235. Purple: 0.470.). 

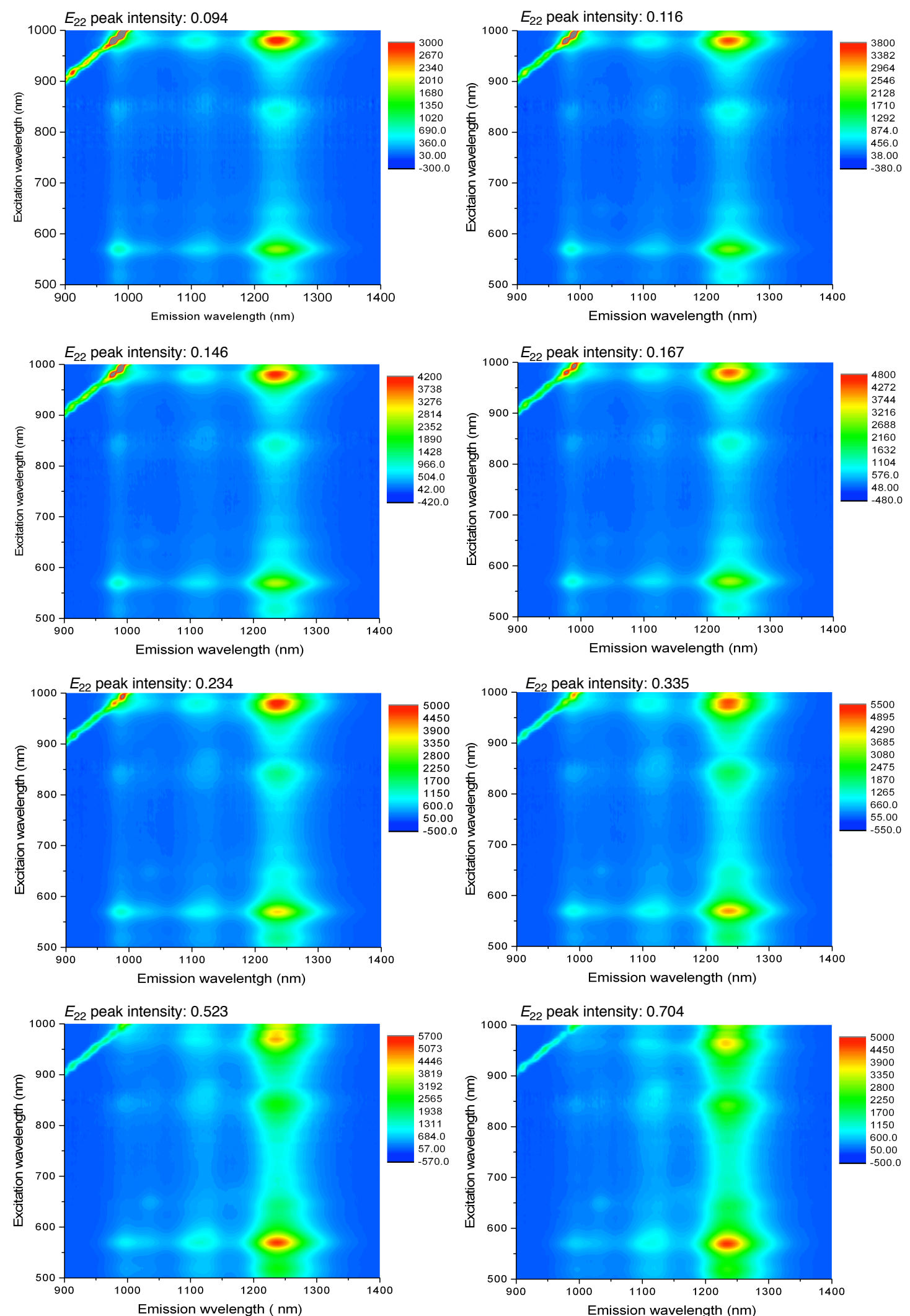

Figure S10 Fluorescence contour plots for H-SWNTs-xylyl (0.0315) at various concentration in 0.5 $\mathrm{wt} \% \mathrm{SC}+0.5 \mathrm{wt} \% \mathrm{SDS}+0.0315 \mathrm{wt} \%$ DOC aqueous solution . 

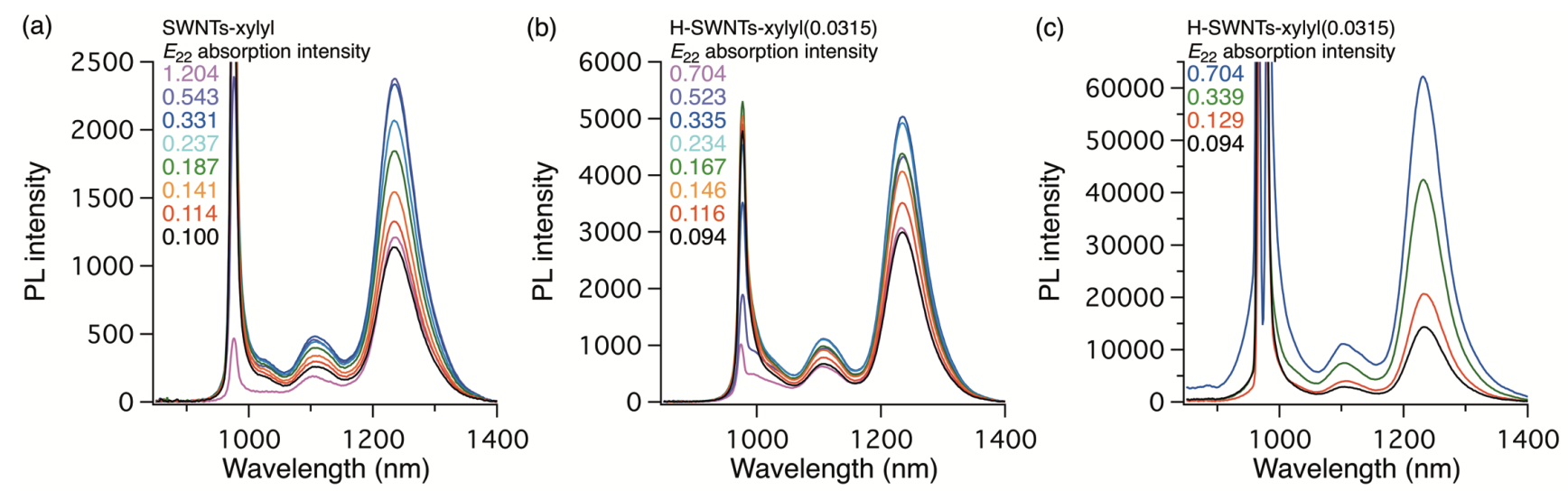

Figure S11 PL spectra ( $\lambda_{\text {ex }}=980 \mathrm{~nm}$ ) of (a) SWNTs-xylyl and (b) H-SWNTs-xylyl (0.0315) at various concentrations (as indicated by the $E_{22}$ absorption peak intensities) measured at a 90 angle. (c) PL spectra $(\lambda \mathrm{ex}=980 \mathrm{~nm})$ of H-SWNTs-xylyl $(0.0315)$ at various concentrations (as indicated by the $E_{22}$ absorption peak intensities) measured at a 22.5 angle.

(a)

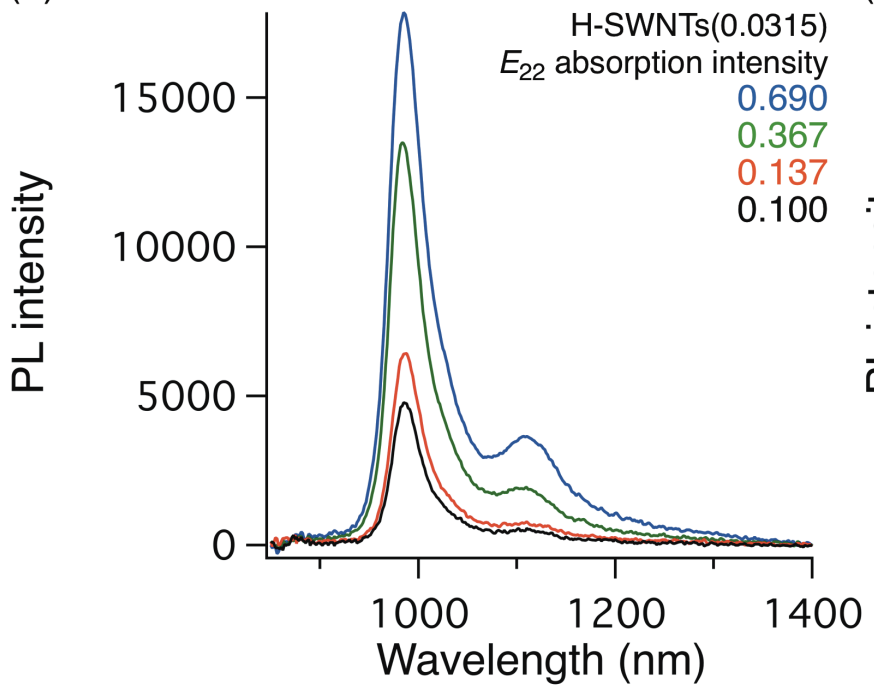

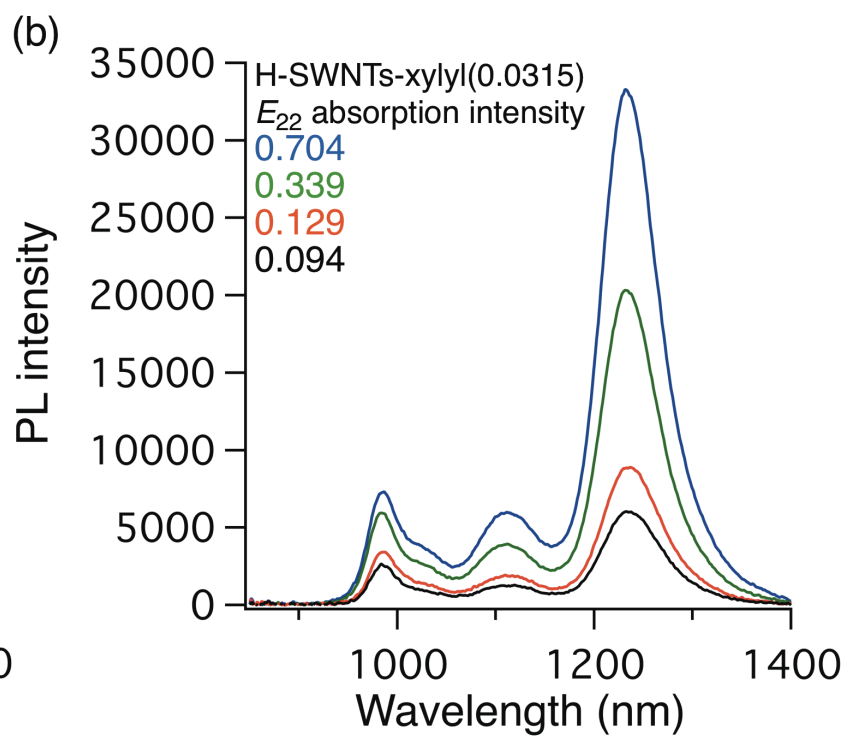

Figure S12 PL spectra $\left(\lambda_{\text {ex }}=580 \mathrm{~nm}\right)$ of (a) H-SWNTs (0.0315) and (b) H-SWNTs-xylyl (0.0315) at various concentrations (as indicated by the $E_{22}$ absorption peak intensities) in $0.5 \mathrm{wt} \% \mathrm{SC}+0.5 \mathrm{wt} \%$ SDS $+0.0315 \mathrm{wt} \%$ DOC aqueous solution measured at a $22.5^{\circ}$ angle relative to the excitation light. 

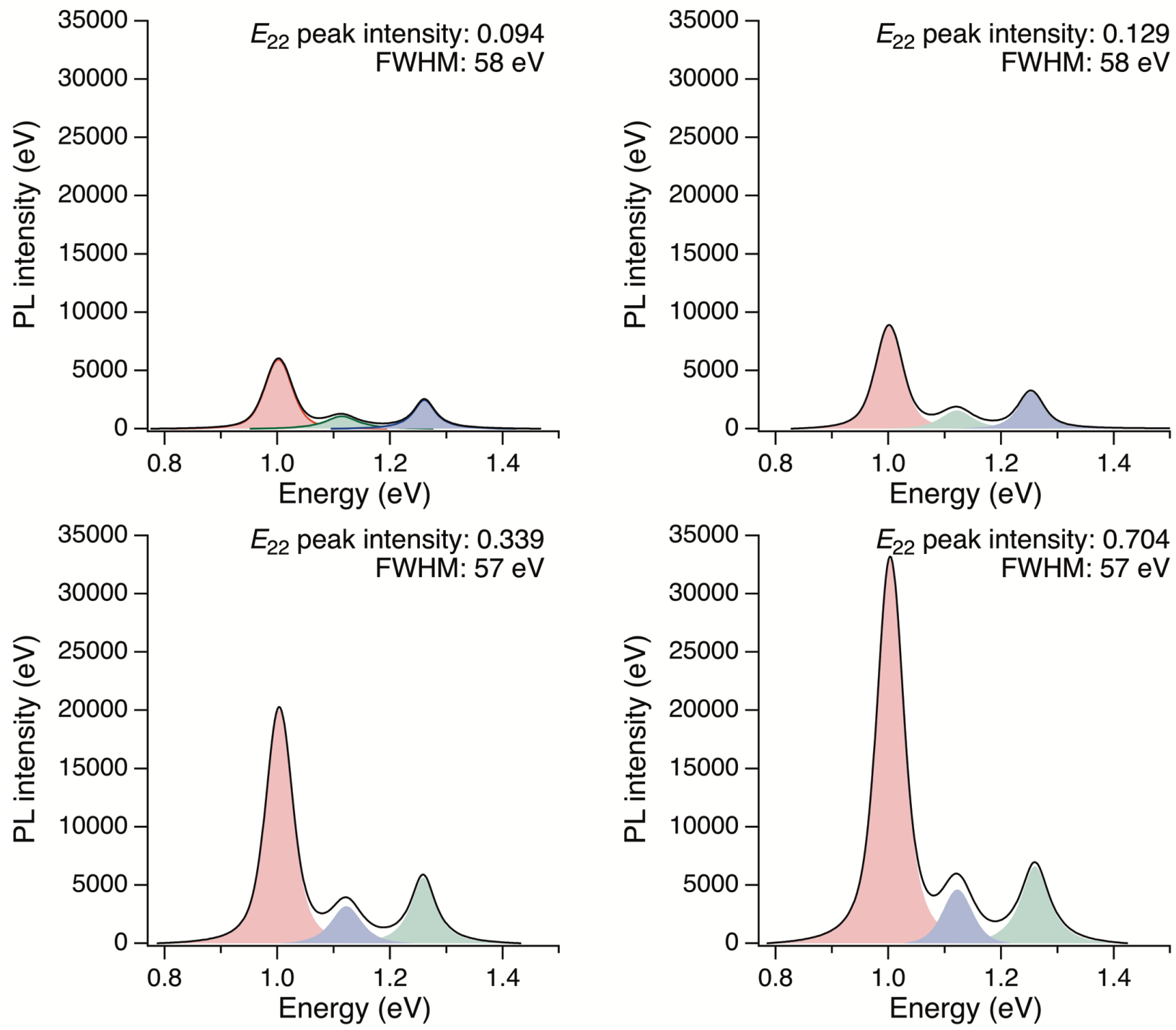

Figure S13 PL spectra ( $E_{22}$ excitation) of H-SWNTs-xylyl (0.0315) (black line) at various concentrations (as indicated by the $E_{22}$ absorption peak intensities) at a $22.5^{\circ}$ angle relative to the excitation light and the corresponding Voigt curve fitting (colored areas). The FWHM values of $\mathrm{E}_{11}{ }^{* *}$ PL peak (red) estimated from the peak fitting. 


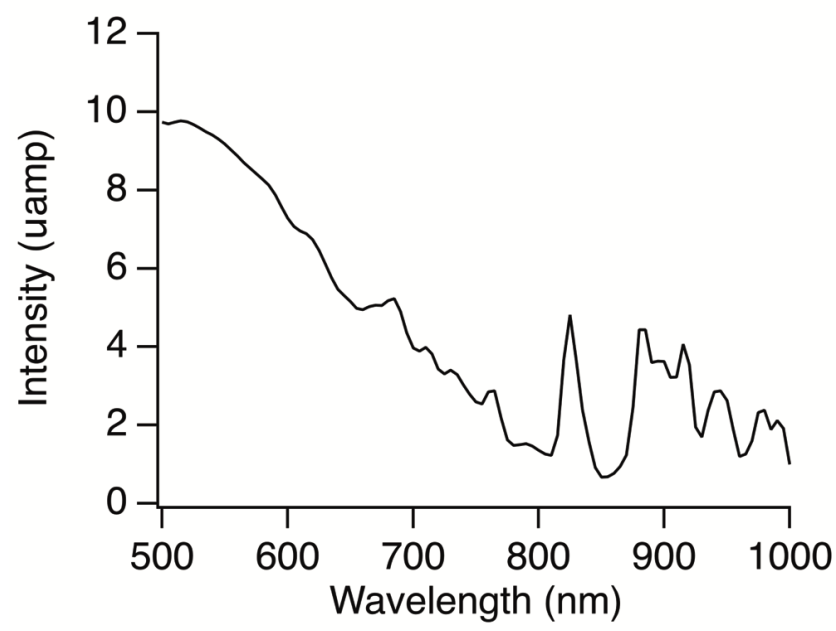

Figure S14 Bright line spectra of a xenon lamp as an excitation source for the PL measurement.

Table S1. $E_{22}$ absorption and $E_{22}$ CD peak wavelength of separated SWNTs.

\begin{tabular}{lccc}
\multicolumn{1}{c}{ SWNTs } & $\begin{array}{c}E_{22} \text { absorption } \\
\text { peak }(\mathrm{nm})\end{array}$ & $\begin{array}{c}E_{22} \text { CD peak } \\
(\mathrm{nm})\end{array}$ & dispersant \\
\hline $\begin{array}{l}\text { SWNTs (0.029) } \\
\text { SWNTs (0.0315) }\end{array}$ & 572 & 574 & SDS, SC, DOC \\
$\begin{array}{l}\text { SWNTs-xylyl } \\
\text { (0.029) }\end{array}$ & 571 & 573 & SDS, SC, DOC \\
SWNTs-xylyl & 570 & 572 & SDS, SC, DOC \\
$\begin{array}{l}(0.0315) \\
(6,5) \text { SWNTs }\end{array}$ & 570 & 572 & SDS, SC, DOC \\
$(11,-5)$ SWNTs & 578 & 578 & flavin mononucleotide ref 1 \\
& 583 & 583 & flavin mononucleotide ref 1
\end{tabular}

Ref 1: Wei, X.; Tanaka, T.; Hirakawa, T.; Yomogida, Y.; Kataura, H., Determination of enantiomeric purity of single-wall carbon nanotubes using flavin mononucleotide. J. Am. Chem. Soc. 2017, 139, 16068-16071. 